\title{
Aggressive Maneuvering of Autonomous Aerial Vehicles: A Human-Centered Approach
}

\author{
M. Piedmonte \\ Autonomous Vehicle Control Group \\ The Charles Stark Draper Laboratory, Inc. \\ Cambridge, MA 02139-3563
}

\author{
Eric Feron \\ Laboratory for Information and Decision Systems \\ Department of Aeronautics and Astronautics, MIT \\ Cambridge, MA 02139
}

\begin{abstract}
Unmanned aerial robots such as autonomous helicopters can perform very aggressive maneuvers that will be very useful in challenging operating conditions. In the absence of detailed models for the vehicle's behavior during those maneuvers, an approach aimed at learning those maneuvers directly from human pilots is proposed. The approach is illustrated for several maneuvers in a simulated environment.
\end{abstract}

\section{Introduction and motivation}

Over the past few years, small helicopters have become the object of intensive investigations as requirements have matured for autonomous air vehicles. Future autonomous vehicle operations in urban and battlefield environments will require extremely agile airborne platforms to perform their missions in dynamic environments. Although some systems based on small helicopters have already demonstrated autonomous operation [1], they display quite modest behavior when compared to the performances obtained from similar platforms by experienced radio-control (R/C) pilots executing aerobatic maneuvers. There are a large number of aggressive flight maneuvers, such as end-over-end forward or reverse flight, extended hovering flight while inverted in ground effect, pop-ups, flips and barrel rolls which are well beyond current autonomous flight capabilities. Enabling these autonomous maneuvers would considerably increase the operational value of these machines, especially in very cluttered or constrained environments such as cities.

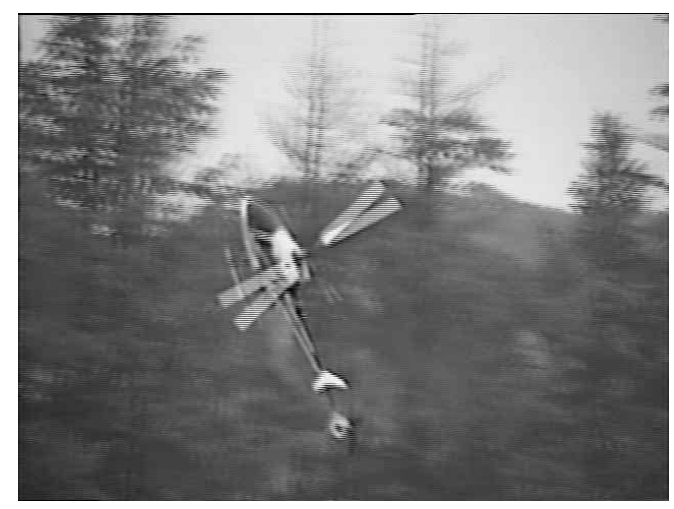

Figure 1: Small R/C helicopter aggressive maneuvers
Several additional factors do contribute to substantial increases in flight performance for autonomous rotorcraft. First, the computational power of on-board flight computers keeps increasing while their size, price, and power requirements decrease. Second, reliable instrumentation both inertial measurement systems and global navigation satellite systems- is shrinking in size and price.

Several approaches exist to enable aggressive maneuvering of platforms such as small autonomous helicopters. Many authors consider model-based approaches whereby the basic dynamics of the vehicle is represented by ordinary differential equations (the equations of motion) $[2,3,4,5]$. In this paper, we propose an intuitive approach to the aggressive control of aerial vehicles, whereby acrobatic maneuvers are learned from human pilots flying remotely controlled machines. Previous work on intuitive control includes, for example, the work of Raibert [6], Pratt and Pratt [7], Crawford and Sastry [8], where the authors use human intuition to derive control strategies for hopping, walking and diving robots. Intuitive control is particularly appealing in the case of aggressive maneuvering of small autonomous aerial vehicles, because (i) most aggressive maneuvers are very difficult to perform automatically, (ii) humans have gathered a large body of intuitive knowledge about these maneuvers and (iii) maneuvers can be documented very precisely with appropriate sensors. Moreover, mathematical helicopter models are well documented only for small attitude angles [9]: At high attitude angles, the dynamics of these systems is uncertain and nonlinear, so that a control design relying too much on a given aircraft mathematical model may not be able to guarantee actual vehicle stability, leading to vehicle divergence and possible crash. On the contrary, aggressive maneuvers recorded from human pilots are by definition feasible. They may then be used towards achieving several tasks: First, they provide feasible reference trajectories that may be used experimentally to explore vehicle dynamics at extreme attitudes without compromising vehicle safety. Second, these experimentally recorded trajectories may directly be used to build a high level model of helicopter dynamics in the form of a "maneuver library", that may be used for higher-level tasks such as path planning without having to perform extensive low-level model identification first. From a control systems perspective, learning control strategies from expert pilots may be seen as a closed-loop 
system identification problem [10], where both the closedloop controller dynamics (the pilot) and the plant closedloop dynamics (the helicopter) are simultaneously identified. As a consequence, not only does the project aim at identifying helicopter dynamics, but also it aims at identifying proven human strategies to control this system.

This paper is organized as follows: First, a description of the experimental setup is provided both for simulated and real flight tests environments. Initial identification results based on closed-loop maneuvering of simulated and real helicopters by a professional R/C pilot are then discussed. It is shown that many aggressive maneuvers may be described in terms of the concatenation of "elemental" maneuvers. Then an initial aggressive maneuvering architecture is introduced, using an existing, operational control architecture developed at Draper Laboratory. This architecture includes coarsely controlled aggressive maneuvers followed by a closed-loop maneuver "recovery" to a trimmed condition. A difficult autonomous maneuver is then simulated.

\section{Experimental Setup}

The goal of the experimental setup was to identify the aggressive maneuvers flown by pilots on small actual and simulated R/C helicopters. For this purpose, the Xcell-60 helicopter platform (shown in Figure 2) was chosen. This platform is very popular among $\mathrm{R} / \mathrm{C}$ pilots, and is capable of very aggressive maneuvers. The simulated environment was a high-level-of-detail model of the Xcell-60 helicopter. The model includes full helicopter dynamics, such as rigid-body, aerodynamic, rotor and servo dynamics. This simulation environment has been in use at Draper Laboratory and MIT since 1996.

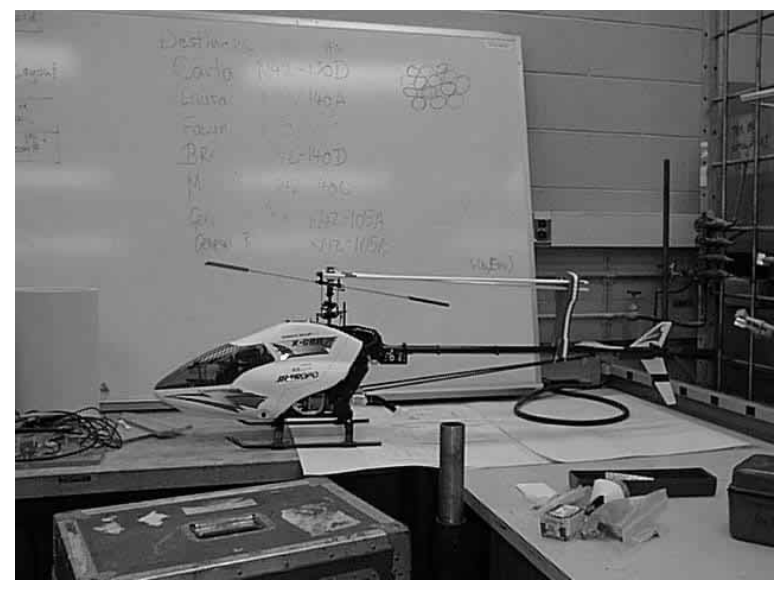

Figure 2: Xcell-60 helicopter

It was originally developed to simulate other helicopters and was modified to include inertial and aerodynamic coefficients specific to the Xcell-60. The model was then fine-tuned using information provided by the $\mathrm{R} / \mathrm{C}$ pilot during test flights. A full validation of the nonlinear simulated helicopter is not available yet, because it requires input-output information on the helicopter state, which was not available at the time this paper was written. Nonlinear helicopter model identification is an area of current research [9].
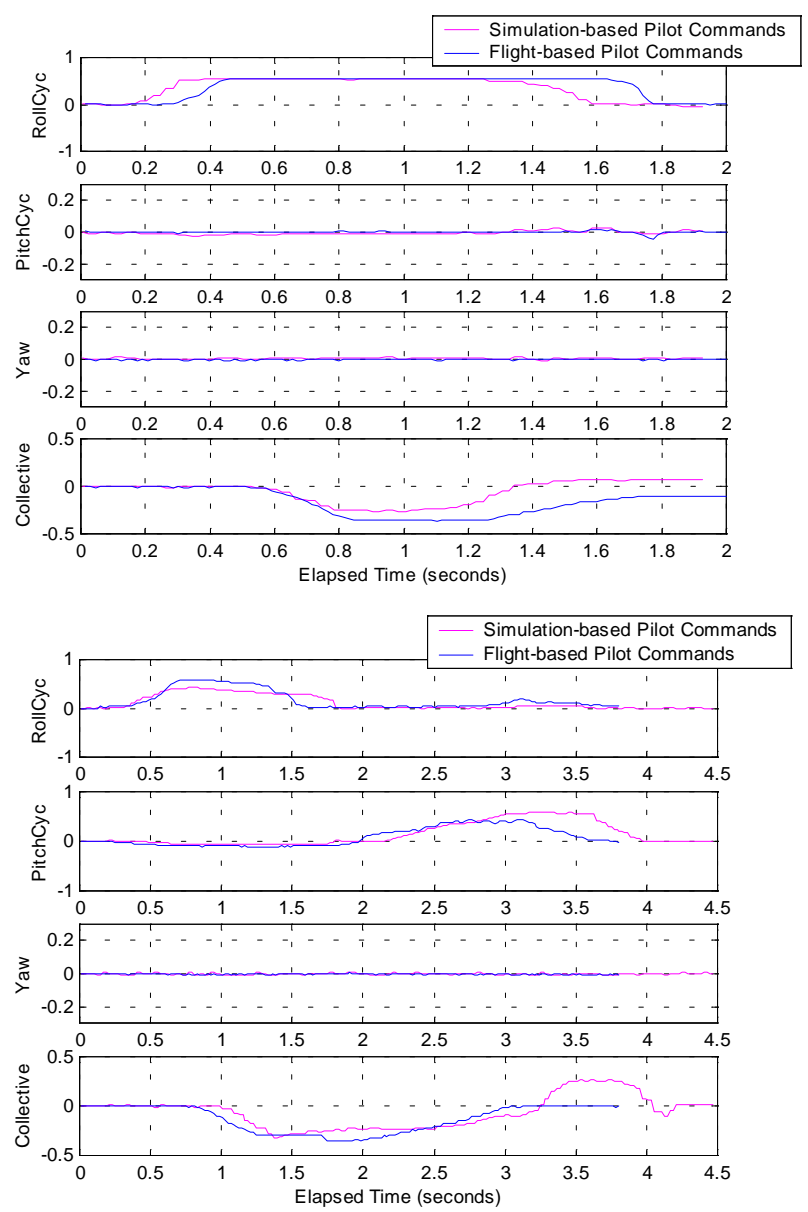

Figure 3: experimental recorded pilot inputs in actual and simulated environments. Top: Barrel roll. Bottom: Split-S.

A professional pilot flew the actual and simulated helicopter, using the same R/C transmitter. When flying the real helicopter, only pilot inputs were digitally recorded, while helicopter state was partially captured on standard video equipment. Acquiring more complete helicopter output information requires much more extensive hardware development efforts, currently under way. Several maneuvers, such as barrel rolls, stall turns, loops, flips and split-S, were then performed by the pilot. The same pilot then flew those maneuvers in the simulated environment. The simulation environment includes high-resolution graphics displayed on a large screen, so that the pilot's view of the helicopter was similar to actual flight conditions, except for helicopter noise, which was absent from the simulation. When using the simulated environment, complete input and helicopter state information was recorded. 

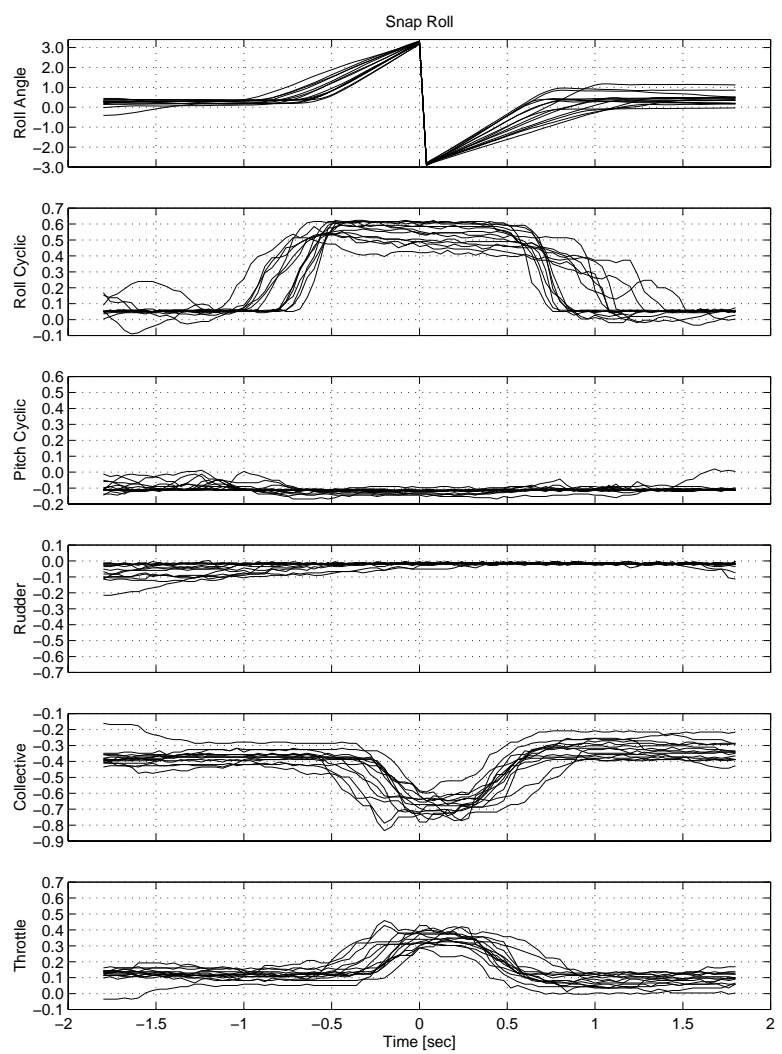

Figure 4: Aggressive maneuvers are often repeatable

The rationale behind these experiments is as follows: Comparing pilot inputs in simulated environments with pilot inputs in actual environments provides a simple and efficient sanity check on whether significant differences exist between pilot aggressive maneuvering strategies in actual and simulated environments. A simulated environment then allows to record full information about the closed-loop pilot/helicopter system at low cost and under controlled wind and visibility conditions.

\section{Observations and maneuver identification}

\subsection{Comparison of actual vs. simulated pilot inputs}

Pilot inputs in simulated and actual environments were recorded for several maneuvers. Figure 3 shows instances of pilot inputs during a barrel roll (the helicopter rolls 360 degrees about its longitudinal axis) and during a split-S: Starting from straight and level flight, the helicopter rolls 180 degrees about its longitudinal axis and then performs half a loop (downwards), resulting in an overall U-turn. Figure 3 shows that the pilot commands for the high-fidelity simulation are quite similar to the pilot commands used while flying the actual helicopter. In both cases, significant variability in maneuver duration was observed. Also, the use of collective seems to differ in real and simulated environments when performing the end of the split-S maneuver; after examination of several maneuvers, this variability turns out not to be correlated with the type of environment (real or simulated) used. Based on these observations, the high level-of-detail simulation, although not formally and fully validated yet, was considered able to capture the most significant features of pilot control strategies when performing aggressive maneuvers.

\section{2 Maneuver identification}

Several maneuvers were recorded and identified during the flight tests in the simulated environment. It was apparent that maneuvers indeed can be differentiated by type (barrel rolls, flips, etc), and that each maneuver type is quite predictable in terms of its sequencing and duration. For example, Figure 4 shows pilot input sequences for several barrel rolls, all of which display the same general characteristics. For all recorded maneuvers, the observed pilot input sequences essentially consist of piecewise constant functions.
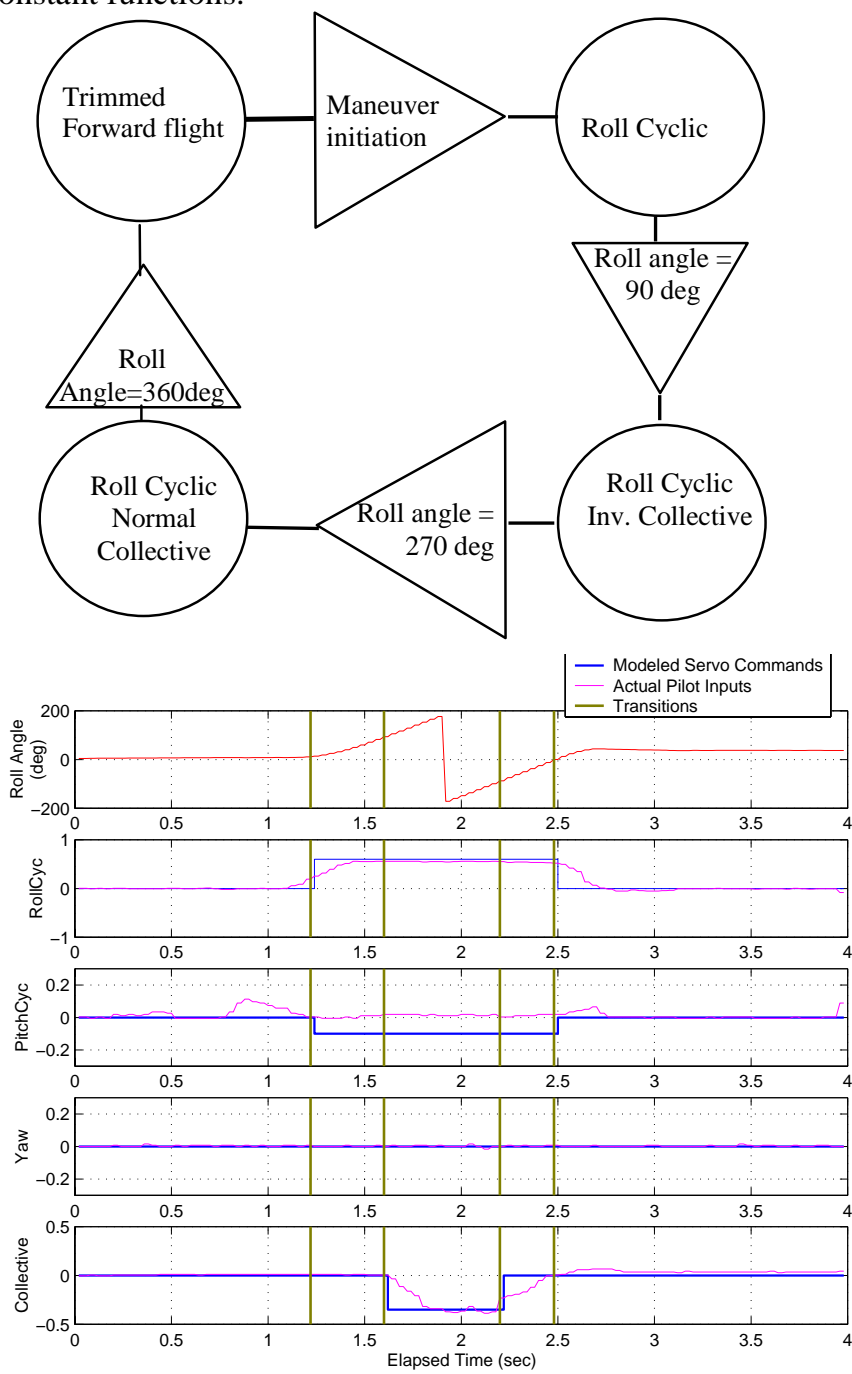

Figure 5: Experimentally determined barrel roll maneuver 
Although the structure of these maneuvers is effectively quite simple, simultaneous observation of state and input signals indicates that pilot control input transitions are more likely to be triggered by specific vehicle state transitions, or might be more easily and robustly encoded as such, resulting in a closed-loop, rather than open-loop maneuver execution. Shown in Figure 5 and 6 are specific state and control time histories for a barrel roll and a split-S maneuver. Considering for example the case of a barrel roll, it was observed that the maneuver is initiated by a pilot step on the roll cyclic command, which causes the aircraft to roll. The collective (helicopter thrust) is inverted as soon as the vehicle reaches 90 degrees roll angle and remains so until the roll angle reaches 270 degrees, at which point it reverts to normal. A flip maneuver is executed likewise, with the pitch cyclic command replacing the roll cyclic command.

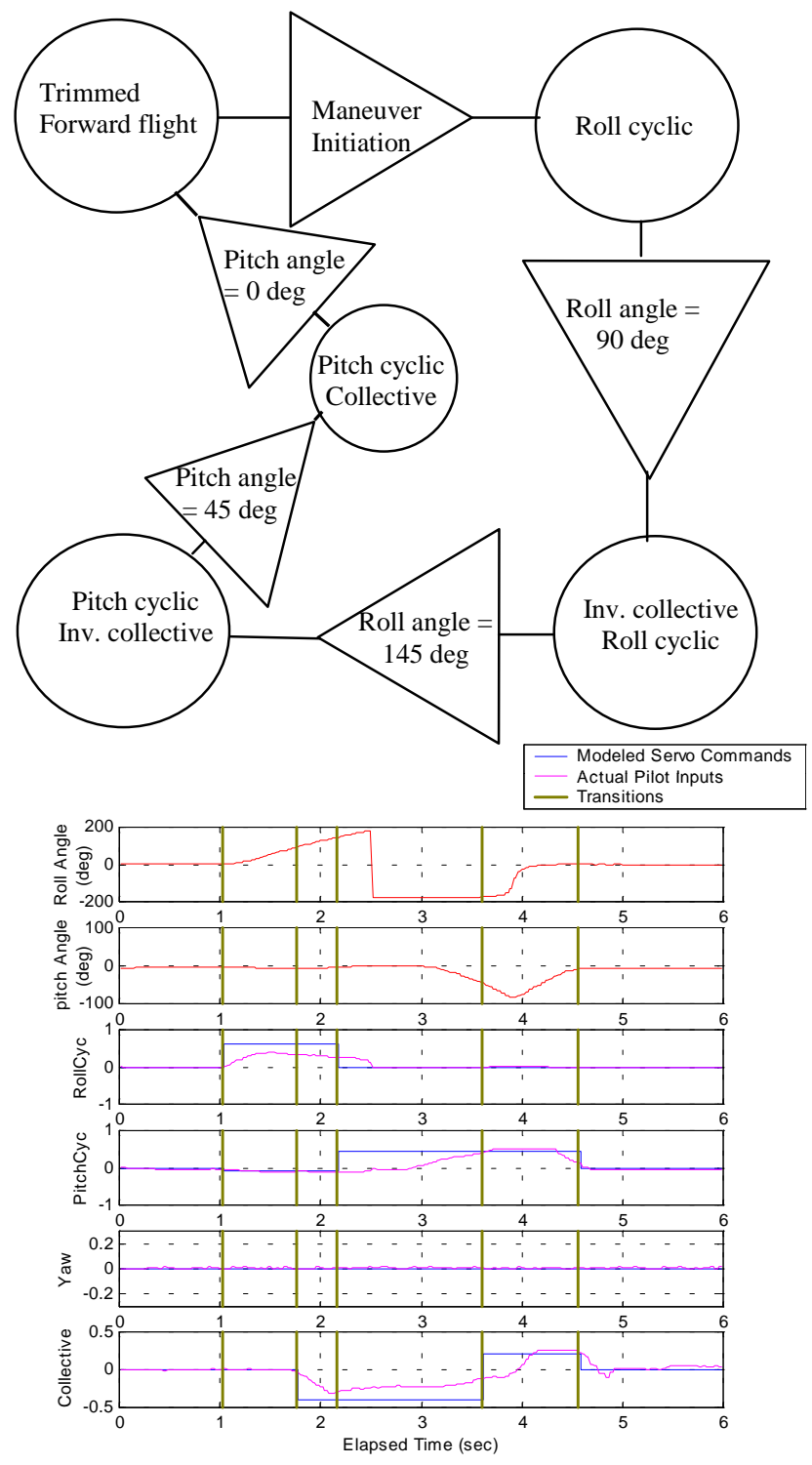

Figure 6: Autonomous Split-S architecture.
The split-S maneuver is more complex, but follows the same logic. Based on these observations, it appears reasonable to code these aggressive maneuvers as finite state machines, where states represent constant control inputs and input transitions are triggered by specific state transitions, also shown in Figure 5 and 6.

\section{Aggressive maneuver architecture design}

\subsection{Implementation on current autonomous architecture}

As a first step towards achieving autonomous aggressive flight, the aggressive maneuver strategies found in the previous section were implemented on the architecture sketched in Fig. 7 and described in detail in [1]. This architecture is operational in the sense that it is routinely used on Draper Laboratory's existing autonomous platforms (two instrumented Bergen helicopters). The existing guidance function generates position, heading, and velocity commands that are sent as reference inputs to the inner-loop feedback control system, currently based on PID loops. These commands are based on the current operational mode of the helicopter, including ground mode, runup mode, takeoff mode, waypoint hover mode, waypoint through mode, track hover mode, track through mode and waypoint land mode.

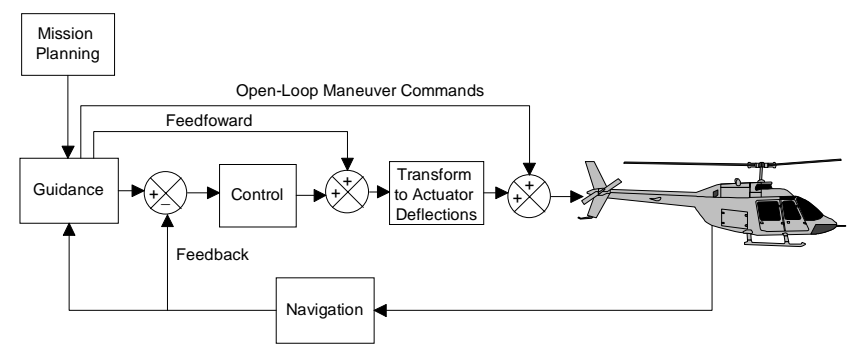

Figure 7: Autonomous Helicopter Supervisory Control Block Diagram.

For the purpose of this project, an aggressive maneuvering mode was added the guidance function. When a maneuver is initiated, the feedback control loop, originally designed for flight conditions near hover is turned off and the maneuver logic described in Paragraph 3.2 is executed instead. Once the maneuver is executed and the vehicle returns to trimmed flight, the original guidance function is turned on again. Experiments show that this is sufficient to recover vehicle control in trim state.

\subsection{Future implementations}

The implementation approach described in Section 4.1 will be useful during early flight tests because it requires only minimal changes to a functional autonomous architecture. Although architecture redesign is a very important and time- 
consuming effort, it will be a necessary step to fully exploit the potential of the aggressive maneuvers identified in this paper. In particular, higher-level robotics applications such as path planning in dynamic environments will require proper and feasible maneuver sequencing. The experimental observations made during this project, combined with previous and current research efforts [1,2,3] indicate that an attractive option is to introduce a maneuver-level, finite-state automaton representation of helicopter dynamics in the control architecture. This automaton is not to be mistaken for those introduced earlier in this paper for the purpose of describing individual maneuvers. The maneuver-level automaton, represented in Fig. 4 (right) and introduced in [2], considers aggressive maneuvers $\left(\mathrm{M}_{1}, \mathrm{M}_{2}, \ldots\right)$ as transitions between trim states (which might be a steady turn, hover, or forward flight).

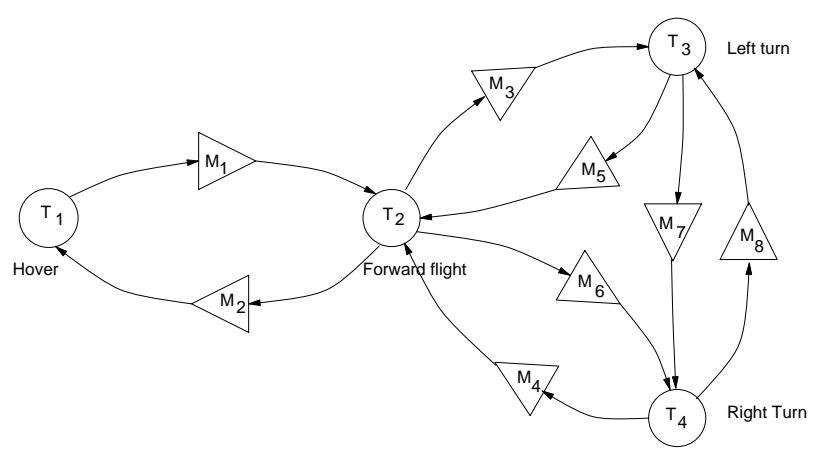

Figure 8: Maneuver-level automaton for autonomous helicopter

Thus a flip, a split-S and a barrel roll would all be transitions from trimmed forward flight onto itself. Trim trajectories, unlike aggressive maneuvers, may last as long as necessary, introducing a hybrid structure into the automaton. This automaton would then represent the helicopter dynamics at the trajectory planning level. Several techniques are currently under investigation for trajectory planning, including randomized search techniques as well as approximate dynamic programming [2,11].

\subsection{Aggressive Maneuver Logic Demonstration}

Using the approach described in the previous paragraph, the high-fidelity simulation of the Xcell-60 helicopter was flown to a forward speed of $35 \mathrm{ft} / \mathrm{sec}$ to then perform a split-S maneuver. This maneuver, usually performed manually on fighter aircraft, consists of half a roll followed by half a flip. It was performed in a simulated urban environment: The autonomous helicopter flies down a narrow street, where a classical U-turn is impossible to perform. Only through a split-S maneuver can the vehicle reverse its direction of flight while not slowing down. The maneuver follows the logic shown in Fig. 6; it is initiated at $t=70 \mathrm{sec}$, ends at $t=74$ sec. Fig. 9 shows a picture of the high-level-of-detail helicopter model heading downwards as it concludes the split-S maneuver and eventually reverses direction of flight.
The state histories show the maneuver being executed, followed by a closed loop control recovery to straight and level flight (past $t=74 \mathrm{sec}$ ).
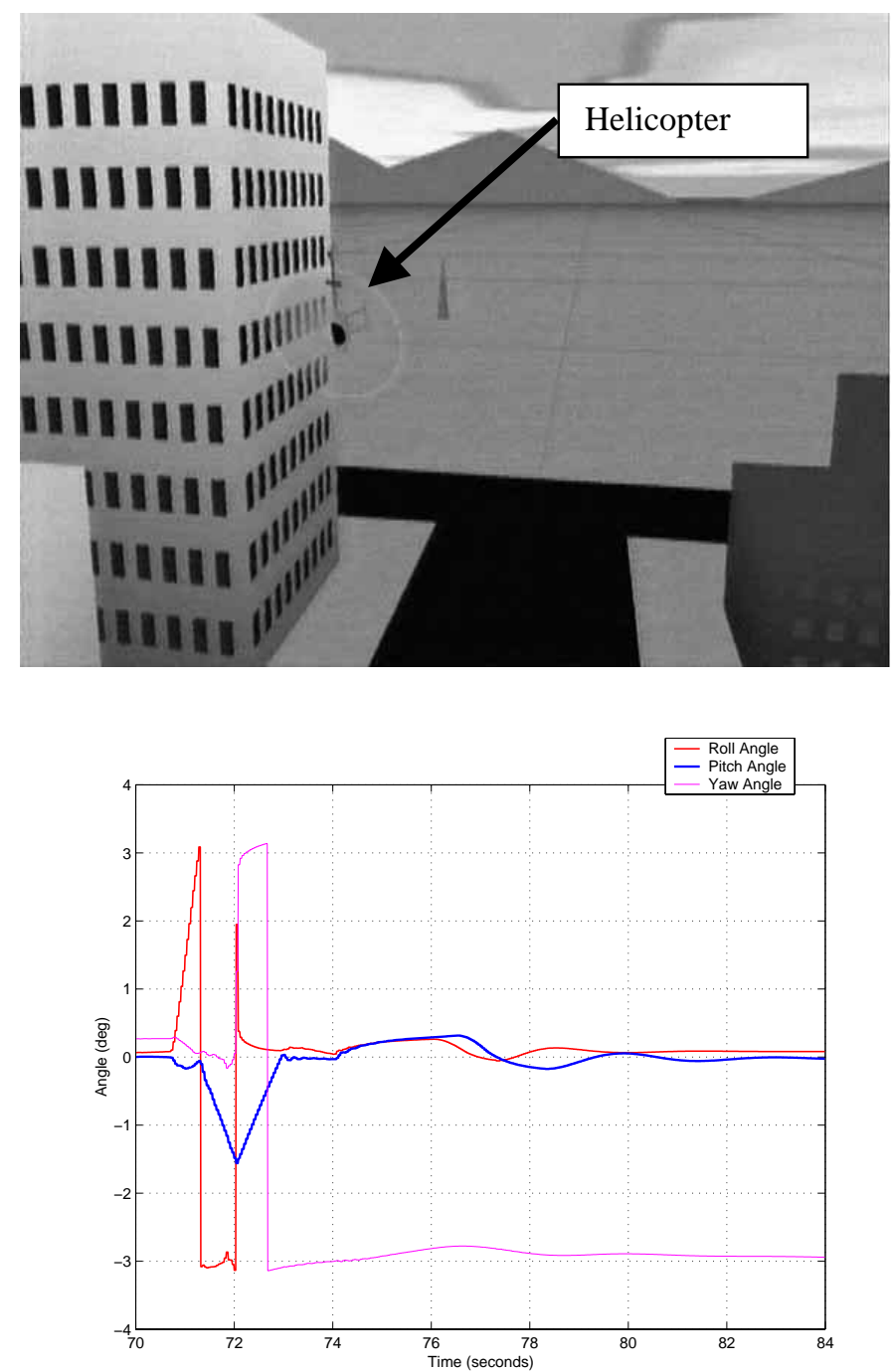

Figure 9: Autonomous split-S maneuver demonstration (simulated environment).

\section{Conclusions and extensions}

The experimental and simulation work performed so far has indicated that aggressive trajectory generation based on recorded human maneuvers is possible and can be automated with piecewise constant control sequences where control amplitude transitions are driven by specific plant state transitions. These maneuvers may be controlled using simple closed-loop trim trajectory recovery.

Extensions to this work include identification and implementation of aggressive maneuvers on an actual small- 
sized rotorcraft. Initial flight tests indicate a significant qualitative similarity between pilot inputs and resulting helicopter behavior in simulated environments and on the real machine. An instrumented flight test program is currently under way at Draper laboratory and MIT. The goal of this program is to record not only pilot inputs but also helicopter states when performing acrobatic maneuvers. This data collection effort will enable a full validation of the helicopter simulation model used in this paper. Further flights will be attempted with full on-board automation. Previous flight tests have revealed that available payload on the Xcell-60 helicopter is sufficient to carry a small on-board computer and appropriate navigation equipment while performing aggressive maneuvers.

\section{Acknowledgements}

The authors would like to thank Brent Appleby, Munther Dahleh, Paul Debitetto, Emilio Frazzoli, Paul Eremenko, Sean George, Alex Lob, Rami Mangoubi, Marc McConley and Scott Rasmussen for their participation in this project. They would also like to thank the reviewers of this paper for their thorough comments. This project was supported by DARPA Contract Number F30602-96-C-0288, Draper Laboratory IR\&D DL-H-505334 and the Office of Naval Research under a Young Investigator Award.

\section{Bibliography}

[1] C. Sanders, P. Debitetto, E. Feron, H.-F. Vuong and N. Leveson. Hierarchical Control of Small Autonomous Helicopters. IEEE Conf. on Decision and Control. December 1998.

[2] E. Frazzoli, M. A. Dahleh and E. Feron. A Hybrid Control Architecture for Aggressive Maneuvering of Autonomous Helicopters, IEEE Conf. On Dec. and Control, December 1999.

[3] D.H. Shim, T.J. Koo, F. Hoffmann, and S. Sastry. A comprehensive study study of control design for an autonomous helicopter. In $37^{\text {th }}$ IEEE Conf. on Decision and Control, 1998.

[4] T.J. Koo and S. Sastry. Output tracking control design of a helicopter model based on approximate linearization. In $37^{\text {th }}$ IEEE Conf. Decision and Control, 1998.

[5] M.J. Van Nieuwstadt and R. M. Murray. Real-time trajectory generation for differentially flat systems. International Journal of Robust and Nonlinear Control, 8(11):995-1020, september 1998.

[6] M. H. Raibert. Legged Robots That Balance. MIT Press, Cambridge, MA, 1986.

[7] J. Pratt and G. Pratt. Intuitive Control of a Planar Bipedal Walking Robot. In Proc. 1998 Int. Conf. Robotics and Automation. Belgium.

[8] L.S. Crawford and S. Shankar Sastry. Biological Motor Control for a Planar Diver. In proc. IEEE Conf. Dec. and Control, 1995.
[9] S. Kim and D. Tilbury. Mathematical Modeling and Experimental Identification of a Model Helicopter. AIAA paper AIAA-98-4357. August 1998.

[10] P. G. Hamel and J. Kaletka. Advances in rotorcraft system identification. Progress in Aerospace Sciences, 1997.

[11] D. Bertsekas and J. N. Tsitsiklis. Neuro-Dynamic Programming. Athena Scientific, 1996.

\section{Appendix: Attitude angles}

The following conventions were used to describe helicopter attitude angles: Roll and yaw angles are between 0 and 360 degrees, modulo 360 degrees. Pitch angle is between -90 degrees and +90 degrees. 